\title{
Non-proportional size scaling of strength of concrete in uniaxial and biaxial loading conditions
}

\author{
G. Qian ${ }^{1}$ (D) | J. Zhai ${ }^{2} \mid$ Z. $\mathrm{Yu}^{2} \mid$ W.-S. Lei $^{3}$ (D) | W. Wu $\mathrm{Wu}^{4,5}$
}

\author{
${ }^{1}$ State Key Laboratory for Nonlinear \\ Mechanics (LNM), Institute of Mechanics, \\ Chinese Academy of Sciences, Beijing \\ 100190, China \\ ${ }^{2}$ School of Materials Engineering, \\ Shanghai University of Engineering \\ Science, 333 Long Teng Rd, Shanghai \\ 201620, China \\ ${ }^{3}$ Applied Materials, Inc., 974 East Arques \\ Avenue, Sunnyvale, CA 94085, USA \\ ${ }^{4}$ Institute of Advanced Structure \\ Technology, Beijing Institute of \\ Technology, Beijing 100081, China \\ ${ }^{5}$ Beijing Key Laboratory of Lightweight \\ Multi-functional Composite Materials and \\ Structures, Beijing 100081, China
}

\section{Correspondence}

W. -S. Lei, Applied Materials, Inc., 974

East Arques Avenue, Sunnyvale, CA

94085, USA.

Email: leiws2008@gmail.com

\begin{abstract}
A procedure for non-proportional size scaling of the strength of concrete based on the weakest-link statistics is proposed to synchronize strength data from specimens of different geometries and different loading modes. The procedure relies on proportional size scaling of strength to determine the parameters of the statistical model and often on finite element analysis to calculate the coefficient of the equivalent strength. The approach for non-proportional size scaling is capable to synchronize the uniaxial strength data of concrete from uniaxial tensile specimens and 3-point bending specimens, or the biaxial tensile strength data of circular plates in different loading mode. The nontransference of the uniaxial strength data to the biaxial strength data is unclear in its mechanism but possibly due to the variation of statistical distribution of microcracks with stress states in different specimens.
\end{abstract}

\section{KEYWORDS}

concrete, microcrack, non-proportional size scaling, size effect, statistical model, strength

\section{1 | INTRODUCTION}

The strength of concrete is a basic mechanical property for integrity assessment of large-scale concrete structures such as dams, bridges, and nuclear power plant structures. The strength of concrete material has 2 distinctive characteristics namely, size effect and random variation. The size effect refers to the inverse correlation between the nominal strength of concrete structures and their geometrical dimensions. The random variation of strength describes the large scatter of strength values measured from a group of nominally identical specimens under same loading conditions. These attributes bring about significant complexity and challenges to the effort on the structural integrity design against failure. Because it is often not affordable or practical to assess the strength and failure behavior by directly testing the full-scale structures, it is much appealing to infer or predict the risk of failure of a large-sized structural component under complex loading conditions from the strength data of small-sized laboratory specimens. This is known as size scaling of strength. Size scaling includes proportional

Nomenclature: $\mathrm{P}, \mathrm{P}(\mathrm{V})$, cumulative failure probability of a solid; $\mathrm{V}$, volume of fracture process zone (FPZ); $\delta \mathrm{V}$, differential volume element; $\mathrm{V}_{0}$, average volume occupied by each microcrack; $\mathrm{p}\left(\sigma, \mathrm{V}_{0}\right)$, fracture probability of a volume element $\mathrm{V}_{0}$ under stress $\sigma$; a, microcrack size; $\mathrm{a}_{\text {max }}$, the maximum microcrack size; $S$, fracture strength of a volume element $V_{0}$; $f(a)$, probability density functions of microcracks with respect to a; $g(S)$, probability density functions of microcracks with respect to $\mathrm{S}$; $\sigma_{\mathrm{th}}$, threshold strength; $N(V)$, number of microcracks in a volume $\mathrm{V}$; m, Weibull modulus; $\sigma_{0}$, Weibull scale parameter; $\sigma_{N}$, nominal fracture strength; $\sigma_{u}$, scale parameter, $\sigma_{u}=\sigma_{0} / k ; h\left(\sigma_{N}\right)$, a function of $\sigma_{N} ; k$, coefficient of equivalent strength, $0<\mathrm{k} \leq 1 ; \xi$, equivalent strength, $\xi=k \cdot\left(\sigma_{N}-\sigma_{t h}\right) ; \mathrm{F}$, fracture load 
scaling and non-proportional scaling. As described in detail earlier, ${ }^{1}$ the proportional scaling concerns the scalability of size effect on strength for a set of geometrically similar structures under nominally same loading conditions (loading mode and loading speed), eg, flexural strength measurement via 3-point bending of prismatic beams with different sizes and loading spans as shown in Figure 1. The non-proportional scaling deals with the transferability of strength data from specimens of 1 given geometry at 1 loading mode to specimens of another geometry at a same or different loading mode, eg, the relationship between the flexural strength of a prismatic beam in 3-point bending and the strength of beams, columns, slabs, and plates in any concrete frame structure. Figure 2 shows examples of specimens with different geometries and loading modes for non-proportional scaling.

Size effect on brittle and quasibrittle fracture has been tackled with both deterministic methods and statistical approaches. ${ }^{1-5}$ The randomness of strength is attributed to the inherent stochastic distribution of defects in concrete in terms of their spatial location, orientation, geometrical shape, and size. This consensus justifies the adoption of statistical approach for size scaling of concrete strength. On the one hand, the weakest link postulate has been commonly adopted as the foundation for statistical approaches to brittle fracture and size scaling $^{1,4-9}$; on the other hand, as analyzed in detail in Lei, ${ }^{10,11}$ there is a long-standing misrepresentation ${ }^{4,5,7-9}$ of the basic weakest-link formulation for brittle fracture, which further leads to a misinterpretation ${ }^{4,5,7,8}$ of Weibull statistics. $^{6}$ This is illustrated in Figure 3. In brief, the previous work by Lei ${ }^{10,11}$ has emphasized the following important aspects of the weakest-link formulation for brittle fracture:

(1) The basic weakest-link formulation for brittle fracture depends on the specific assumption of the spatial distribution of microcracks in a material;

(2) Historically, both the uniform spatial distribution and the Poisson spatial distribution have been adopted;

(3) The assumption of mutual non-interaction of microcracks is needed to develop the weakest-link formulation;

(4) The uniform spatial distribution is compatible with the assumption of mutual non-interaction of microcracks, which encompasses Weibull statistics as its special case;

(5) The Poisson spatial distribution conflicts with the assumption of mutual non-interaction of microcracks, resulting in the difficulty to develop the weakest-link formulation;
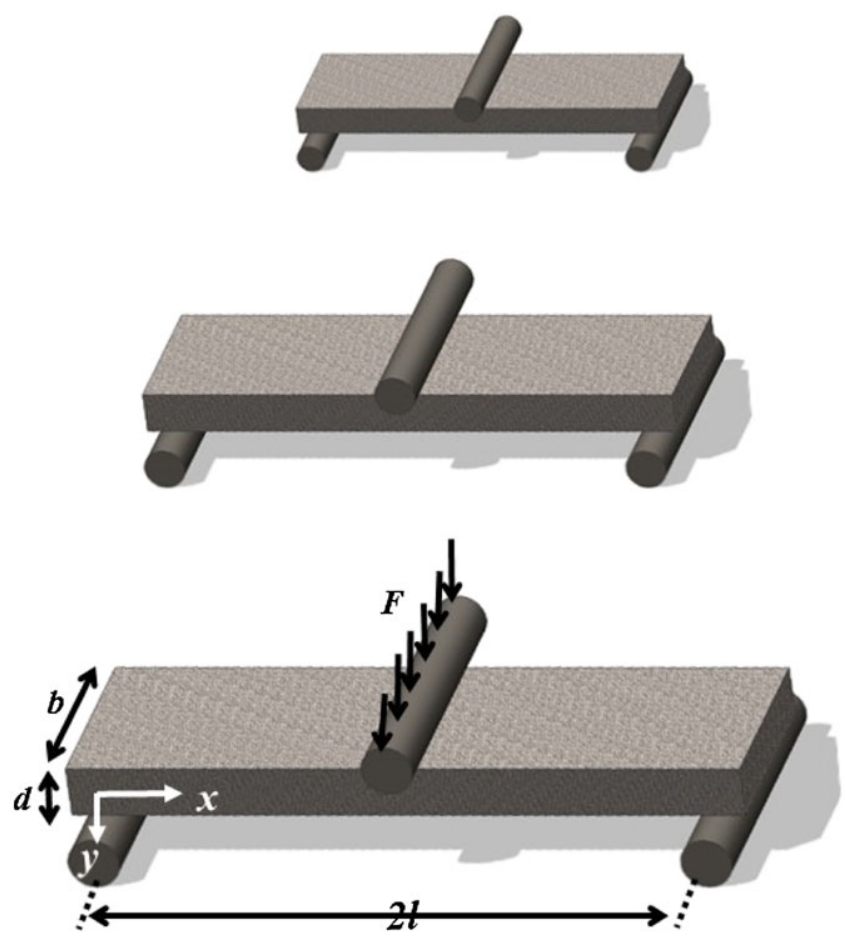

FIGURE 1 Proportional size scaling of strength in 3-point flexural specimens [Colour figure can be viewed at wileyonlinelibrary.com]

(6) Recent work $^{12}$ proposed a more generic weakestlink formulation based on the assumption of power-law spatial distribution of microcracks, which makes the uniform spatial distribution as its special case;

(7) Weibull statistics ${ }^{6}$ was developed based on the uniform spatial distribution of microcracks; The conventional practice of using a power-law microcrack size distribution to interpret Weibull statistics is invalid, which can be stretched to mimic the 2-parameter Weibull statistics, but fails to formulate the 3-parameter Weibull statistics.

To be more specific, as highlighted in Figure 3, many researches falsely adopted the following weakestlink formulation for the cumulative probability of failure ${ }^{4,5,7-9}$ :

$$
P(V)=1-\exp \left[-\int_{V} p\left(\sigma, V_{0}\right) \cdot \delta V / V_{0}\right]
$$

where $\mathrm{P}=\mathrm{P}(\mathrm{V})$ is the cumulative probability of failure of a solid, $\mathrm{V}$ is the volume of fracture process zone (FPZ). Within FPZ, the equivalent stress that controls fracture initiation, $\sigma_{e}=\sigma$, which is a function of 3 principal stresses $\left(\sigma_{1}, \sigma_{2}, \sigma_{3}\right)$ and microcrack orientation, exceeds the critical fracture strength $\mathrm{S}$, ie, $\sigma_{e}=\sigma \geq S$, 
(A)
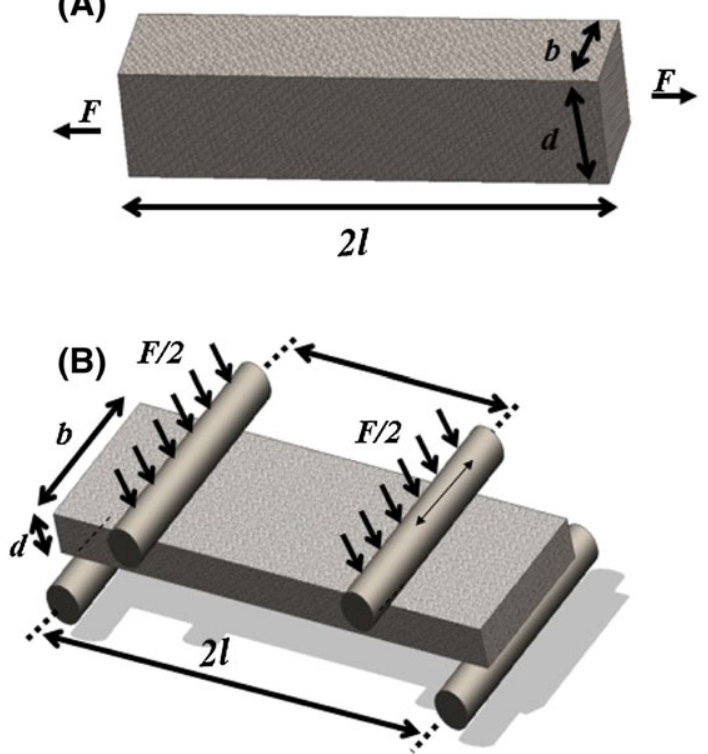
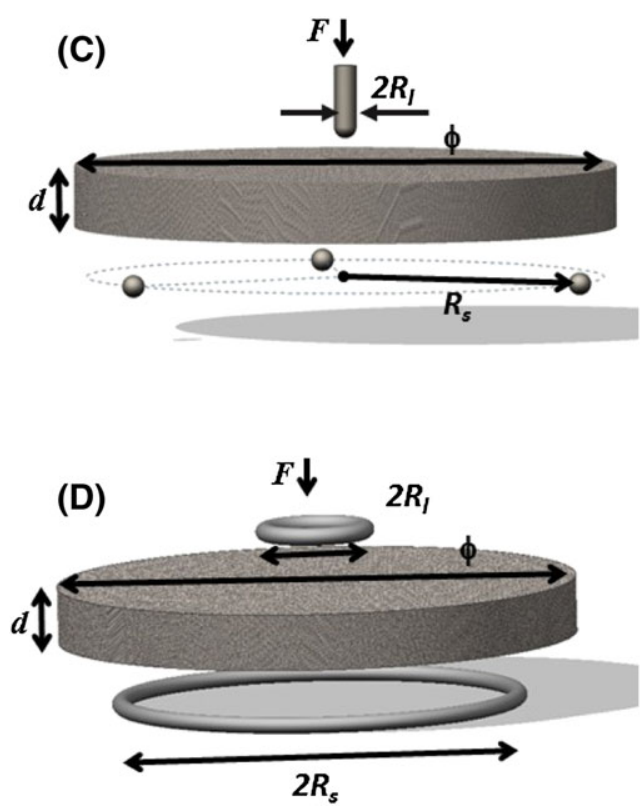

FIGURE 2 Non-proportional size scaling of strength in specimens of different geometries and loading modes [Colour figure can be viewed at wileyonlinelibrary.com]

$\delta \mathrm{V}$ is a differential volume element, $\mathrm{V}_{0}$ is the average volume occupied by each microcrack. $\mathrm{p}\left(\sigma, \mathrm{V}_{0}\right)$ is the fracture probability of a volume element $\mathrm{V}_{0}$ due to the unstable propagation of a microcrack embedded therein under stress $\sigma_{e}=\sigma$. Under the maximum principal stress fracture criterion $\left(\sigma_{e}=\sigma_{1}=\sigma \geq S\right), \mathrm{p}\left(\sigma, \mathrm{V}_{0}\right)$ is given by

$$
p\left(\sigma, V_{0}\right)=\int_{\sigma_{t h}}^{\sigma} g(S) d S=\int_{a}^{a_{\max }} f(a) d a
$$

where $g(S)$ and $f(a)$ are the probability density functions (PDFs) of microcracks with respect to fracture strength (S) and microcrack size (a), respectively, $a_{\max }$ is the maximum microcrack size $\left(0 \leq \mathrm{a} \leq a_{\max }\right), \sigma_{\text {th }}$ is the threshold strength $\left(\sigma_{t h} \leq S<\infty\right)$. According to the Griffith law, S. $\sqrt{a}=$ Const.

Equation 1 is problematic. As the basic formulation for the weakest-link statistics, Equation 1 should be selfconsistent within the whole variable ranges of volume $V_{0} \leq V<\infty$ and probability $0 \leq \mathrm{p}\left(\sigma, V_{0}\right) \leq 1$. However, substitution of $V=V_{0}$ in Equation 1 leads to

$$
P\left(V_{0}\right)=1-\exp \left[-p\left(\sigma, V_{0}\right)\right] \neq p\left(\sigma, V_{0}\right)
$$

While substitution of $\mathrm{p}\left(\sigma, V_{0}\right)=0$ and $\mathrm{p}\left(\sigma, V_{0}\right)=1$ in Equation 1 yields

$$
P(V)=\left\{\begin{array}{cc}
0 & \left(p\left(\sigma, V_{0}\right)=0\right) \\
1-\exp \left(-V / V_{0}\right) \neq 1 & \left(p\left(\sigma, V_{0}\right)=1\right)
\end{array}\right.
$$

Obviously, both Equations 3 and $4 \mathrm{~b}$ make no sense.

Equation 1 was derived by assuming the spatial distribution of microcracks to follow either the Poisson distribution law or the uniform distribution law (Figure 3). As explained in Lei, ${ }^{11}$ the assumption of Poisson spatial distribution of microcracks is not compatible with the mutual independence of microcracks that underlies the Griffith law relating the strength (S) and size (a) of an arbitrary microcrack. While in the case of a uniform spatial distribution of microcracks, the following inappropriate approximation was introduced to obtain Equation 1:

$$
\ln \left[1-p\left(\sigma, V_{0}\right)\right] \approx-p\left(\sigma, V_{0}\right)
$$

As elaborated in Lei, ${ }^{10}$ Equation 5 is fairly accurate only for $0 \leq p\left(\sigma, V_{0}\right) \leq 0.2$ ! Figure 4 compares the values of $-\ln \left[1-p\left(\sigma, V_{0}\right)\right]$ and $p\left(\sigma, V_{0}\right)$. By definition, the probability of failure $p\left(\sigma, V_{0}\right)$ always obeys $0 \leq p\left(\sigma, V_{0}\right) \leq 1$. Due to both the random size distribution of a microcrack within a volume element $V_{0}$ at an arbitrary spatial location and the arbitrary stress $(\sigma)$ distribution thereat, there is actually no guarantee that $p\left(\sigma, V_{0}\right)$ is always much smaller than 1 at an arbitrary spatial location. In other words, because one cannot justify that $p\left(\sigma, V_{0}\right)$ is always much smaller, Equation 5 is invalid.

The adoption of the problematic Equation 1 further led to a twisted interpretation of Weibull statistics by assuming a power-law distribution of microcrack size (a) as follows,

$$
f(a)=\mu \cdot a^{-\kappa}(\mu>0, \kappa>1)
$$




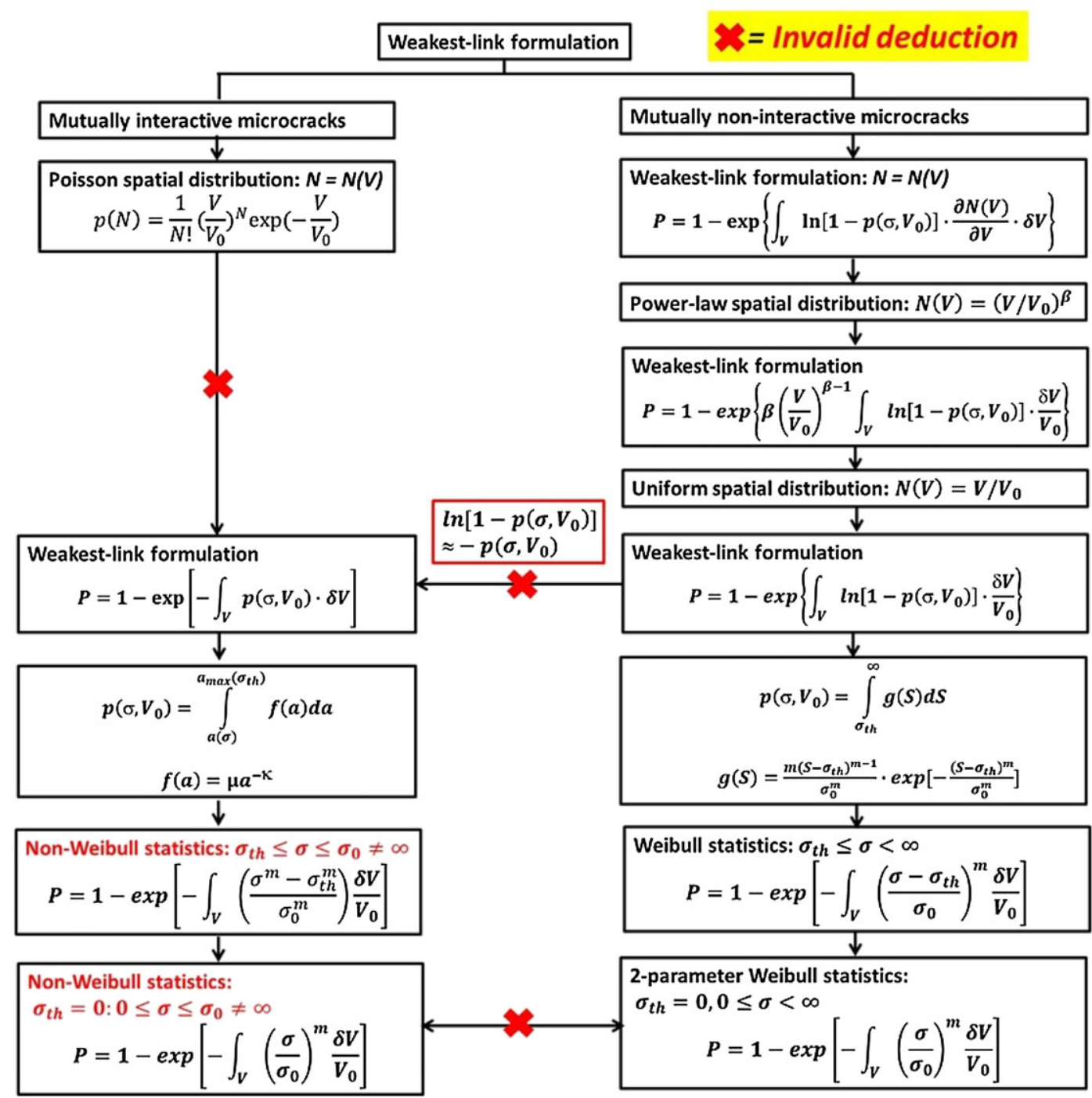

FIGURE 3 Illustration of different weakest-link formulations [Colour figure can be viewed at wileyonlinelibrary.com]

Substitution of Equation 6 in Equation 2 gives

$$
p\left(\sigma, V_{0}\right)=\frac{1}{\kappa-1}\left(a^{\kappa-1}-a_{\max }^{\kappa-1}\right) \stackrel{\sigma \sqrt{a}=\text { Const }}{=} \frac{\sigma^{m}-\sigma_{t h}^{m}}{\sigma_{0}^{m}}
$$

By assuming $a_{\max } \rightarrow \infty$ or $\sigma_{t h}=0$, Equation 7 is reduced to

$$
p\left(\sigma, V_{0}\right)=\frac{a^{\kappa-1}}{\kappa-1}=\left(\frac{\sigma}{\sigma_{0}}\right)^{m} \quad\left(0 \leq \sigma \leq \sigma_{0}\right)
$$

Substitution of Equation 8 in Equation 1 leads to the expression seemingly same as the 2-parameter Weibull statistics but with essential difference in the valid range of stress $\sigma$ :

$$
P(V)=1-\exp \left[-\int_{V}\left(\frac{\sigma}{\sigma_{0}}\right)^{m} \cdot \frac{\delta V}{V_{0}}\right] \quad\left(0 \leq \sigma \leq \sigma_{0}\right)
$$

Note that due to Equation 8, Equation 9 is only applicable to the stress range $0 \leq \sigma \leq \sigma_{0}$, while the 2-parameter Weibull statistics applies to the whole stress range $0 \leq \sigma<\infty$ ! Based on Equation 9, Bazant and coworkers ${ }^{4,5,9}$ mistakenly concluded that Weibull statistics is applicable only to a chain of infinite links (also known as the infinite weakest link model) but not to the finite weakest link model. As we will show below (Figure 3), in fact, the power-law distribution of microcrack size (a) in Equation 6 was not adopted in Weibull's work. ${ }^{6}$

Recently, Lei ${ }^{12}$ proposed the generalized weakest-link formulation for brittle fracture induced by a population of mutually non-interactive microcracks as follows:

$$
P=1-\exp \left\{\int_{V} \ln \left[1-p\left(\sigma, V_{0}\right)\right] \cdot \frac{\partial N(V)}{\partial V} \cdot \delta V\right\}
$$

where $N=N(V)$ is the number of microcracks in a volume $\mathrm{V}$. 


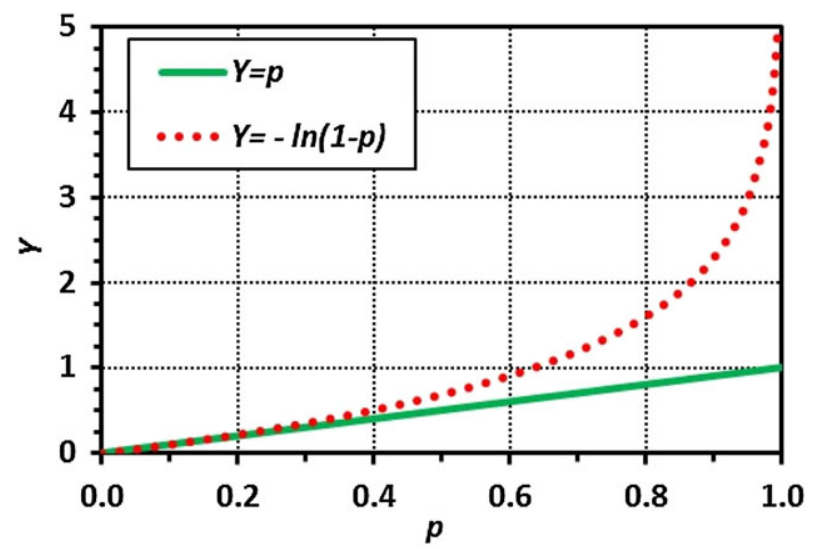

FIGURE 4 The difference between $-\ln \left[1-p\left(\sigma, V_{0}\right)\right]$ and $p(\sigma$, $V_{0}$ ) [Colour figure can be viewed at wileyonlinelibrary.com]

When the number of microcracks in a volume $\mathrm{V}$ takes the power-law function as below:

$$
N=N(V)=\left(\frac{V}{V_{0}}\right)^{\beta} \quad(\beta>0)
$$

Equation 10 reduces to

$$
P=1-\exp \left\{\beta\left(\frac{V}{V_{0}}\right)^{\beta-1} \int_{V} \ln \left[1-p\left(\sigma, V_{0}\right)\right] \cdot \frac{\delta V}{V_{0}}\right\}
$$

According to the first mean value theorem for integrals, Equation 12 is rewritten as

$$
\frac{1}{\beta V^{\beta}} \cdot \ln \left[\frac{1}{(1-P)}\right]=\frac{1}{V_{0}^{\beta}} \cdot \ln \left[\frac{1}{1-p\left(\xi, V_{0}\right)}\right]=\frac{h\left(\sigma_{N}\right)}{V_{0}^{\beta}}
$$

where $\xi$ is a stress value in the range $\sigma_{t h} \leq \xi \leq \sigma_{N}$. In principle, there is $\xi=k \cdot\left(\sigma_{N}-\sigma_{t h}\right)$, with $k$ being a constant $(0<\mathrm{k} \leq 1), h\left(\sigma_{N}\right)$ is a function of the nominal fracture strength $\sigma_{N}$.

Note that $\beta>0$ includes 3 scenarios, namely $\beta=1$, $0<\beta<1, \beta>1$, as exemplified in Lei. ${ }^{12}$ When $\beta=1$, the microcracks are uniformly distributed within a material, Equations 12 and 13 reduce to the following in sequence,

$$
\begin{gathered}
P=1-\exp \left\{\int_{V} \ln \left[1-p\left(\sigma, V_{0}\right)\right] \cdot \frac{\delta V}{V_{0}}\right\} \\
\frac{1}{V} \cdot \ln \left[\frac{1}{(1-P)}\right]=\frac{1}{V_{0}} \cdot \ln \left[\frac{1}{1-p\left(\xi, V_{0}\right)}\right]=\frac{h\left(\sigma_{N}\right)}{V_{0}}
\end{gathered}
$$

Equation 14 can reasonably interpret Weibull statistics. In fact, when $g(S)$ is described by

$$
g(S)=\frac{m}{\sigma_{0}}\left(\frac{S-\sigma_{t h}}{\sigma_{0}}\right)^{m-1} \cdot \exp \left[-\left(\frac{S-\sigma_{t h}}{\sigma_{0}}\right)^{m}\right] \quad\left(\sigma_{t h} \leq S<\infty\right)
$$

substitution of Equation 16 in Equation 2 gives

$$
p\left(\sigma, V_{0}\right)=1-\exp \left[-\left(\frac{\sigma-\sigma_{t h}}{\sigma_{0}}\right)^{m}\right] \quad\left(\sigma_{t h} \leq \sigma<\infty\right)
$$

Consequently, Equations 12 and 14 reduce to

$$
P=1-\exp \left\{-\beta\left(\frac{V}{V_{0}}\right)^{\beta-1} \int_{V}\left(\frac{\sigma-\sigma_{t h}}{\sigma_{0}}\right)^{m} \frac{\delta V}{V_{0}}\right\} \quad\left(\sigma_{t h} \leq \sigma<\infty\right)
$$

$$
P=1-\exp \left\{-\int_{V}\left(\frac{\sigma-\sigma_{t h}}{\sigma_{0}}\right)^{m} \frac{\delta V}{V_{0}}\right\} \quad\left(\sigma_{t h} \leq \sigma<\infty\right)
$$

$$
\text { or } \begin{aligned}
\ln \left[\frac{1}{1-p\left(\xi, V_{0}\right)}\right] & =h\left(\sigma_{N}\right) \\
& =\left(\frac{\sigma_{N}-\sigma_{t h}}{\sigma_{0}}\right)^{m} \quad\left(\sigma_{t h} \leq \sigma_{N}<\infty\right)
\end{aligned}
$$

Equation 20 was actually used by Weibull to deduce Weibull statistics ${ }^{6}$ according to Equation 15, instead of the power-law distribution of microcrack size (a) in Equation 6. Note that in Equation 20, the upper bound of $\sigma_{N}$ extends to infinity $(\infty)$. Moreover, Equation 20 is based on Equation 16. In the case that a different assumption of $\mathrm{g}(\mathrm{S})$ is adopted, the specific expression of $h\left(\sigma_{N}\right)$ in Equation 15 will vary correspondingly.

Equation 13 provides an approach to scaling size effect on strength by establishing the correlation between the compound parameter $\frac{1}{\beta V^{\beta}} \cdot \ln \left[\frac{1}{(1-P)}\right]$ and nominal strength $\sigma_{N}$ of specimens with different sizes. Equation 13 was validated by the strength data of a wide spectrum of quasi-brittle materials including wood, concrete, coal, gamma titanium aluminum alloy, nuclear-grade graphite, and aluminum foam on geometrically self-similar specimens under same loading conditions. Specific to concrete, some initial results show that $\beta=1$ for uniform spatial distribution of microcracks applies. ${ }^{1,12}$ Therefore, this work will resort to Equations 14 and 15.

Equation 15 was successfully applied to the proportional size scaling of strength of concrete. ${ }^{1,12}$ However, the non-proportional size scaling of strength remains a 
big challenge. An earlier work ${ }^{1}$ suggested to tackle this problem by understanding the following 2 aspects:

(1) multiaxial-stress based fracture criteria, and

(2) susceptibility of spatial distribution of microcracks to stress state.

With respect to the fracture criteria, different stressbased microscopic fracture criteria $\left(\sigma_{e}=\sigma \geq S\right)$ have been proposed, with each one corresponding to a specific expression of the effective stress $\sigma_{\mathrm{e}}{ }^{13,14}$ Among them, the maximum tensile principal stress fracture criterion $\left(\sigma_{e}=\sigma_{1}=\sigma \geq S\right)$ is the simplest, so that Equation 2 is applicable to evaluate the fracture probability due to a single micro crack $p\left(\sigma, V_{0}\right)$. For all the other microscopic fracture criteria, Equation 2 is no longer valid, and it is much complicated to evaluate $p\left(\sigma, V_{0}\right)$. As an example, the analytical solution to $p\left(\sigma, V_{0}\right)$ under the normal tensile stress $\left(\sigma_{\mathrm{n}}\right)$ criterion is given in Lei. ${ }^{15}$

With respect to the susceptibility of spatial distribution of microcracks to stress state, because concrete is a quasi-brittle material, microcracks can initiate or grow under external loading. It is thus reasonable to question whether the spatial distribution of microcracks in concrete changes with stress state, eg, from uniaxial tension to biaxial tension.

It calls for significant amount of work to understand these 2 questions for non-proportional size scaling. Before pursuing that path, we believe it is meaningful to evaluate the applicability of the following simplified assumptions:

(1) The maximum principal tensile stress fracture criterion $\left(\sigma_{e}=\sigma_{1}=\sigma \geq S\right)$ applies to different stress states so long as at least 1 tensile stress component exists;

(2) The spatial distribution of microcracks does not change with stress state.

This work will explore the non-proportional size scaling of the strength of concrete based on these 2 simplified assumptions.

\section{2 | PROCEDURE FOR NON- PROPORTIONAL SIZE SCALING}

As stated earlier, regardless of the specific stress state, the spatial distribution of microcracks is assumed the same and the maximum principal tensile stress fracture criterion $\left(\sigma_{e}=\sigma_{1}=\sigma \geq S\right)$ applies. Also, based on the initial results, ${ }^{1,12}$ we will assume that for concrete, the uniform spatial distribution of microcracks $(\beta=1)$ applies.
Therefore, Equations 14 and 15 are adopted for size scaling of concrete strength. This leads to the proposal of the following procedure for non-proportional size scaling, as also shown in Figure 5:

(1) Determination of the empirical expression of $h\left(\sigma_{N}\right)$

According to Equation 15, there exists a master curve that characterizes the unique correlation between the compound parameter $\frac{1}{V} \cdot \ln \left[\frac{1}{(1-P)}\right]$ and nominal strength $\sigma_{N}$ of geometrically similar specimens. For simplicity of discussion, here below $\sigma_{t h}=0$ is assumed. By performing proportional size scaling of strength, the empirical expression of the strength function $h\left(\sigma_{N}\right)$ can be estimated. Once $h\left(\sigma_{N}\right)$ is known, due to Equation 15, we get

$$
p\left(\xi=k \cdot \sigma_{N}, V_{0}\right)=1-\exp \left[-h\left(\sigma_{N}\right)\right]
$$

$$
\text { and, } g\left(\sigma_{N}\right)=\frac{\partial p\left(k \cdot \sigma_{N}, V_{0}\right)}{\partial \sigma_{N}}=h^{\prime}\left(\sigma_{N}\right) \cdot \exp \left[-h\left(\sigma_{N}\right)\right]
$$

Note that $k$ is a constant with $0<k \leq 1$. At this point, the function format of the probability $p\left(\xi=k \cdot \sigma_{N}, V_{0}\right)$ for an arbitrary microcrack or the PDF of microcracks with respect to nominal strength $\sigma_{N}, g\left(\sigma_{N}\right)$, is determined. But until the value of the constant $\mathrm{k}$ is known, the explicit relationship between $p\left(\xi=k \cdot \sigma_{N}, V_{0}\right)$ and $\xi$ cannot be achieved. This is because other geometrically independent model parameters as material constants such as the Weibull scale parameter $\sigma_{0}$ can be still entangled with $k$.

To elaborate the proposed procedure, now assume that the correlation between $\frac{1}{V} \cdot \ln \left[\frac{1}{(1-P)}\right]$ and the nominal strength $\sigma_{N}$ can be approximated by $\frac{1}{V} \cdot \ln \left[\frac{1}{(1-P)}\right] \propto \sigma_{N}^{m}$. According to Equation 15, this suggests that the function $h\left(\sigma_{N}\right)$ fits to the power function in Equation 20 with $\sigma_{t h}=0$. As a result, Equation 19 reduces to

$$
P=1-\exp \left[-\int_{V}\left(\frac{\sigma_{1}}{\sigma_{0}}\right)^{m} \frac{\delta V}{V_{0}}\right]
$$

Equation 23 is rewritten as

$$
\begin{aligned}
1-P & =\exp \left[-\left(\frac{\sigma_{N}}{\sigma_{0}}\right)^{m} \frac{V}{V_{0}} \int_{V}\left(\frac{\sigma_{1}}{\sigma_{N}}\right)^{m} \frac{\delta V}{V}\right] \\
& =\exp \left[-\left(\frac{k \sigma_{N}}{\sigma_{0}}\right)^{m} \frac{V}{V_{0}}\right]
\end{aligned}
$$

Note that the power function in Equation 20 is a simple and often typical expression to describe the function 


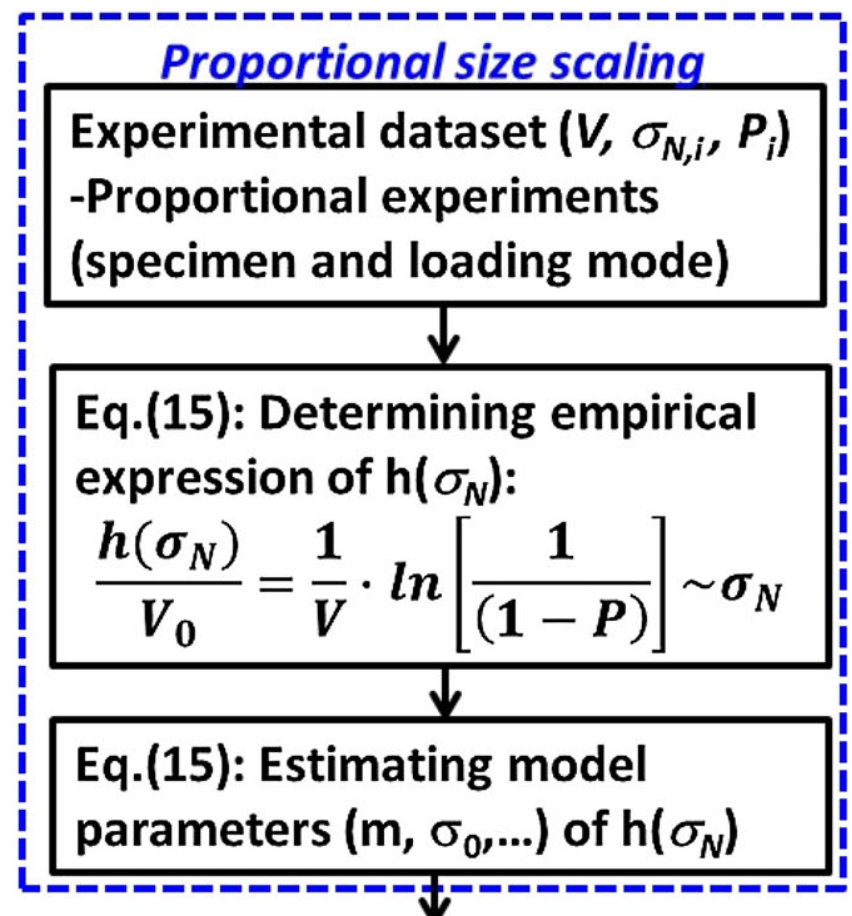

Calculating $k: \xi=k \cdot \sigma_{N}$

$\boldsymbol{p}\left(\xi, V_{0}\right)=1-\exp \left[-\boldsymbol{h}\left(\sigma_{N}\right)\right]$

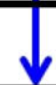

Non-proportional size scaling

$\frac{1}{V} \cdot \ln \left[\frac{1}{(1-P)}\right] \sim \xi=k \cdot \sigma_{N}$

个

\section{Experimental dataset $\left(V, \sigma_{N, i}, P_{i}\right)$ -Non-proportional experiments (specimen and/or loading mode)}

FIGURE 5 Flow chart of non-proportional size scaling procedure [Colour figure can be viewed at wileyonlinelibrary.com]

$h\left(\sigma_{N}\right)$. Due to the different types of microcrack distributions, other expressions are possible.

(2) Estimation of some model parameters

Once the empirical expression of the function $h\left(\sigma_{N}\right)$ is determined, it is feasible to estimate at least some model parameters. For example, Equation 24 can be further expressed in 3 different formats:

$$
\begin{gathered}
\operatorname{Ln}\left\{\frac{1}{V} \operatorname{Ln}\left[\frac{1}{(1-P)}\right]\right\}=m \operatorname{Ln}\left(\sigma_{N}\right)-\operatorname{Ln}[V_{0} \cdot \underbrace{\left(\sigma_{0} / k\right)^{m}}_{\sigma_{u}}] \\
\frac{1}{V} \operatorname{Ln}\left[\frac{1}{(1-P)}\right]=m \operatorname{Ln}(\underbrace{k \cdot \sigma_{N}}_{=\xi})-\operatorname{Ln}\left(\sigma_{0}^{m} V_{0}\right) \\
\frac{1}{k^{m} \cdot V} \operatorname{Ln}\left[\frac{1}{(1-P)}\right]=m \operatorname{Ln}\left(\sigma_{N}\right)-\operatorname{Ln}\left(\sigma_{0}^{m} V_{0}\right)
\end{gathered}
$$

with

$$
\begin{gathered}
k=\left[\frac{1}{V} \int_{V}\left(\frac{\sigma_{1}}{\sigma_{N}}\right)^{m} d V\right]^{\frac{1}{m}} \\
\sigma_{u}=\sigma_{0} / k
\end{gathered}
$$

These 3 equivalent expressions in Equation 25a,b,c can be used for different purposes. Specifically, Equation 25a is used for proportional size scaling. This leads to the estimated values of $\mathrm{m}$ and the compound parameter $\sigma_{u}$ (defined in Equation 27), while the pure material property $\sigma_{0}$ and the dimensionless coefficient $k$ are to be determined.

\section{(3) Computation of parameter $k$}

$k$ reflects the effect of specimen geometry and loading mode on the equivalent strength $\xi=k \sigma_{N}$. Numerical calculation is often needed to determine the values of $k$ for specimens of dissimilar geometries or different loading modes, unless an analytical solution of $k$ is available. The value of $k$ remains the same for geometrically similar specimens under a same loading mode. Therefore, as stated earlier, the correlation between $\frac{1}{V} \cdot \ln \left[\frac{1}{(1-P)}\right]$ and the nominal strength $\sigma_{N}$ based on Equation 15 is sufficient for proportional size scaling of strength regardless of the value of $k$. However, the nonproportional scaling of strength demands the value of $k$ in order to establish the correlation between $\frac{1}{V} \cdot \ln \left[\frac{1}{(1-P)}\right]$ and the mean strength $\xi=k \cdot \sigma_{N}$, which is transferrable between different specimen geometries and different loading mode.

For the example given in Equation 24, the value of $k$ is calculated according to Equation 26. 


\section{(4) Non-proportional size scaling}

With the numerically determined values of $k$ for different specimens as input, according to Equation 15, the experimental strength data can be synchronized by correlating $\frac{1}{V} \cdot \ln \left[\frac{1}{(1-P)}\right]$ and $\xi=k \cdot \sigma_{N}$ to enable non-proportional scaling of strength.

Equation $25 \mathrm{~b}$ can be used for non-proportional size scaling by correlating the compound parameter $\frac{1}{V} \operatorname{Ln}\left[\frac{1}{(1-P)}\right]$ and the equivalent strength $\xi=k \sigma_{N}$. Also, the value of $\sigma_{0}$ is known by Equation 27. Alternatively, the non-proportional size scaling can be also realized via establishing the correlation between the other compound parameter $\operatorname{Ln}\left\{\frac{1}{k^{m} \cdot V} \operatorname{Ln}\left[\frac{1}{(1-P)}\right]\right\}$ and the nominal strength $\sigma_{N}$ according to Equation 25c. In this case, the product $k^{m} \cdot V$ can be taken as an effective volume.

In the following, we will adopt some published strength data of concrete to conduct non-proportional scaling based on Equation 25c.

\section{3 | CASE STUDIES OF NON- PROPORTIONAL SIZE SCALING OF CONCRETE STRENGTH}

\subsection{Concrete strength in uniaxial tensile stress state}

The first example includes published strength data of asphalt mixture in 3-point bending of 3 different sized specimens and in uniaxial tension of a given sized specimen by Le, et al. ${ }^{9}$ The nominal average size of taconite aggregates and pit sand is $1.22 \mathrm{~mm}$. The sizes of the 3point bending specimens are (Figure 1): $b=40 \mathrm{~mm}$, $2 l=6 d$, with $d=16.7 \mathrm{~mm}, 28.9 \mathrm{~mm}$, and $50 \mathrm{~mm}$, respectively. The sizes of the uniaxial tensile specimen are (Figure 2A): $b=d=55 \mathrm{~mm}, 2 l=255 \mathrm{~mm}$. The nominal strengths for the 3-point bending specimens $\sigma_{N, B}$ and the uniaxial tensile specimen $\sigma_{N, T}$ are parameterized as:

$$
\begin{gathered}
\sigma_{N, B}=\frac{3 F l}{b d^{2}}=\frac{9 F}{b d} \\
\sigma_{N, T}=\frac{F}{b d}
\end{gathered}
$$

where $\mathrm{F}$ is the fracture load.

The detailed strength data were tabulated in Le et $\mathrm{al}^{9}$ and are represented in Figure 6A, where the ordinate is
(A)

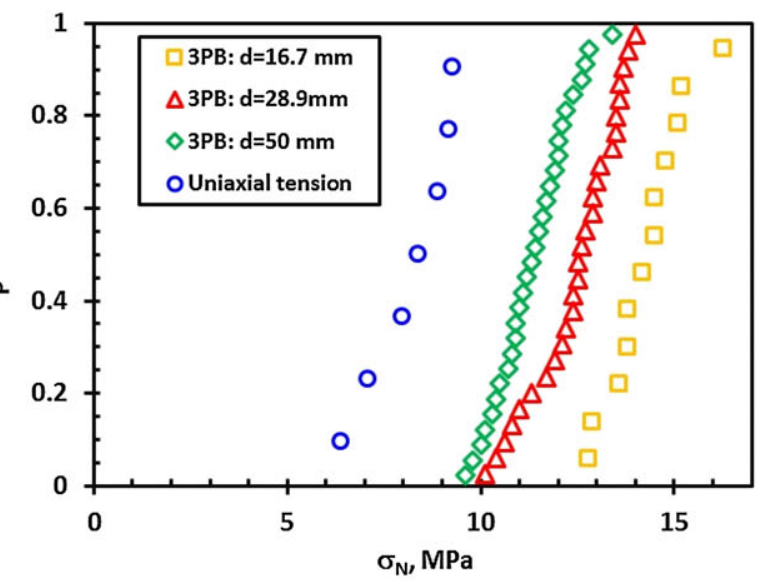

(B)

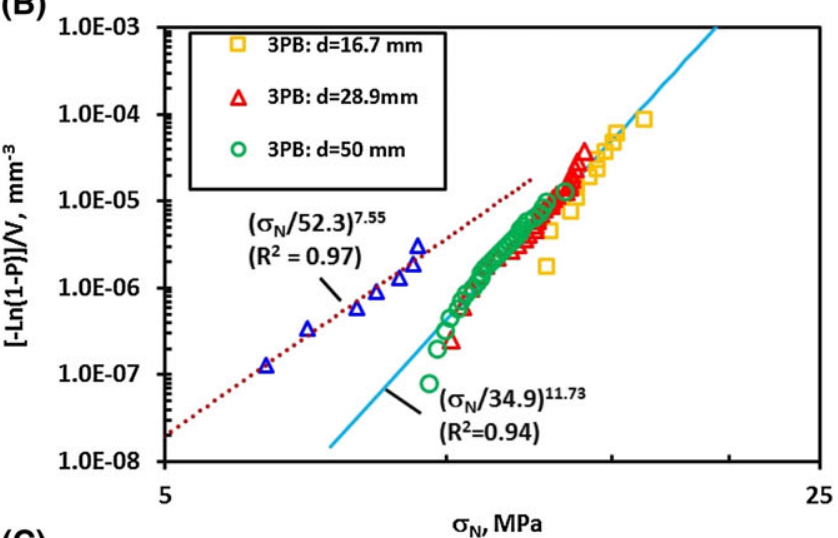

(C)

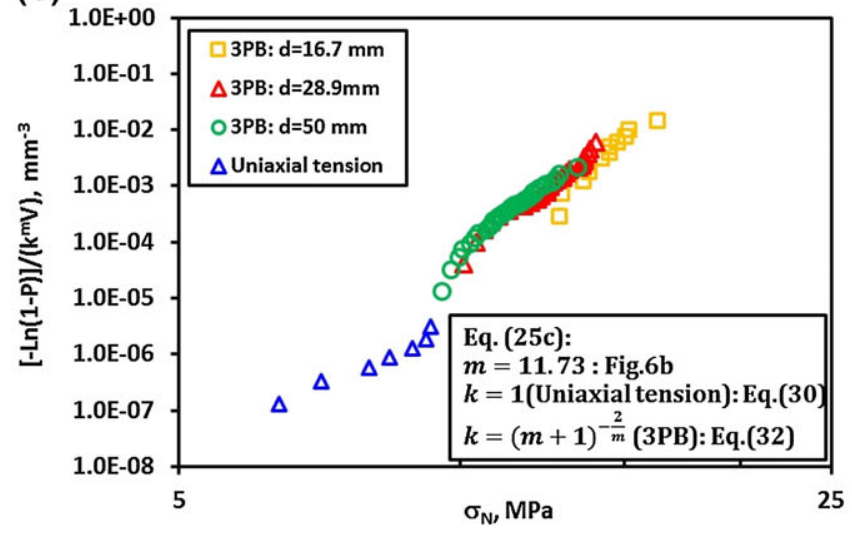

FIGURE 6 Non-proportional size scaling of strength in uniaxial tensile specimens and 3-point bending (3PB) specimens: A, raw experimental strength data ${ }^{16}$; B, proportional size scaling; $C$, nonproportional size scaling with $\mathrm{m}=11.7$ [Colour figure can be viewed at wileyonlinelibrary.com]

the rank probability $\mathrm{P}\left(\sigma_{N, i}\right)=(i-0.3) /(n+0.4)$ corresponding to the $i$-th nominal strength $\sigma_{N, i}$ out of all the $\mathrm{N}$ nominal strength values ranked in an ascending order for a group of given sized specimens. Now, the strength data in Figure 6A are converted to Figure 6B according the proportional scaling relation in Equation 15 or $25 \mathrm{a}$. It shows that the 3 sets of strength data from the 3-point bending specimens fall onto 1 master curve which can be fitted by the power-law with $\mathrm{m}=11.73$. Equation $25 \mathrm{a}$ is 
adopted for linear regression of the experimental data in Figure $6 \mathrm{~B}$ to estimate $\mathrm{m}$. This is the key parameter obtained from the proportional size scaling analysis. This makes it possible to adopt Equation 25b or 25c for nonproportional size scaling analysis. Besides, there are 2 points for caution. First, due to very limited number of data points (only 7) for uniaxial tensile specimens, the estimated value of $m=7.55$ is lower than $m=11.73$ from 70 data points of 3-point bending specimens. Second, the strength data of uniaxial tensile specimens do not fall onto the same master curve for the 3-point bending specimens. This is expected because the value of dimensionless coefficient $k$ in Equations 24-27 is different for the 3-point bending specimens and for the uniaxial tensile specimens. For the uniaxial tensile specimen, it is obvious that

$$
k_{T}=1
$$

Refer to Figure 1, the stress distribution in the 3-point bending specimen is

$$
\sigma_{1}(x, y)=\sigma_{N, B} \cdot \frac{2 x y}{L d}=\sigma_{N, B} \cdot \frac{2 x y}{3 d^{2}}
$$

Substitution of Equation 31 in Equation 26 yields

$$
k_{B}=(m+1)^{-\frac{2}{m}}
$$

The Weibull modulus $m$ is a material parameter. It should be the same for specimens under either uniaxial tension or 3-point bending. Accordingly, with $\mathrm{m}=11.73$ obtained from proportional scaling of 3-point bending specimens in Figure 6B as input together with the values of $k$ in Equations 30 and 32, Figure 6B is transformed into Figure 6 $\mathrm{C}$ according to Equation $25 \mathrm{c}$. It is obvious that all data fall onto a master curve. The "kink" between the data points for 3-point bending and those for uniaxial tension should be due to very limited 7 strength data for uniaxial tension.

\section{2 | Concrete strength in uniaxial and biaxial tensile stress states}

$\mathrm{Zi}$, et $\mathrm{al}^{17}$ studied the size effect on uniaxial and biaxial flexure strength of concrete. The uniaxial flexure strength was measured with the 4-point bending (4PB) test of rectangular beams (Figure 2B). The equi-biaxial flexure strength was assessed with the ASTM C1550 (C1550) flexure test (Figure $2 \mathrm{C}$ ) and the ring-on-ring (RoR) flexure test (Figure 2D) of circular plates. In each case, 3 sets of specimens with different sizes (small, medium, and large) are tested, with 13 specimens per set. The nominal stress $\sigma_{\mathrm{N}}$ is defined as follows:

Four-point bending (4PB) specimen (Figure 2B):

$$
\sigma_{N, 4 P B}=\frac{2 F l}{b d^{2}}
$$

ASTM C1550 (C1550) flexural specimen (Figure 2C):

$\sigma_{N, C 1550}=\frac{3 F(1+v)}{4 \pi d^{2}}\left[1+2 \operatorname{Ln}\left(\frac{R_{s}}{r_{e}}\right)+\left(\frac{1-v}{1+v}\right)\left(\frac{2 R_{s}^{2}-r_{e}^{2}}{2 R_{0}^{2}}\right)\right]$

Ring-on-ring (RoR) flexural specimen (Figure 2D):

$$
\sigma_{N, R o R}=\frac{3 F}{4 \pi d^{2}}\left[2(1+v) \operatorname{Ln}\left(\frac{R_{s}}{R_{l}}\right)+\frac{(1-v)\left(R_{s}^{2}-R_{l}^{2}\right)}{R_{0}^{2}}\right]
$$

where $\mathrm{F}$ is the load, $\mathrm{d}$ is the depth of a $4 \mathrm{~PB}$ specimen or the plate thickness for $\mathrm{C} 1550$ and RoR specimens, $2 l$ and $\mathrm{b}$ are the outer loading span and the thickness of a $4 \mathrm{~PB}$ specimen, $v$ is the Poisson's ratio, $R_{0}=\phi / 2$ is the radius of the circular plate, $R_{S}$ is the distance from

\begin{tabular}{|c|c|c|c|c|}
\hline \multicolumn{2}{|l|}{ Specimen Dimensions, $\mathrm{mm}$} & \multirow{2}{*}{$\begin{array}{c}\text { Small } \\
30\end{array}$} & \multirow{2}{*}{$\begin{array}{l}\text { Medium } \\
48\end{array}$} & \multirow{2}{*}{$\begin{array}{r}\text { Large } \\
75\end{array}$} \\
\hline 4-point flexure (Figure 2B) & $\mathrm{d}$ & & & \\
\hline & $\mathrm{b}$ & 30 & 48 & 75 \\
\hline & $2 l$ & 90 & 144 & 225 \\
\hline & $2 c$ & 30 & 48 & 75 \\
\hline \multirow[t]{4}{*}{ ASTM C1550 (Figure 2C) } & Thickness (d) & 30 & 48 & 75 \\
\hline & $\operatorname{Diameter}(\phi)$ & 262 & 420 & 657 \\
\hline & Radius to support $\left(\mathrm{R}_{\mathrm{S}}\right)$ & 125 & 200 & 312.5 \\
\hline & Radius of loading area $\left(\mathrm{R}_{l}\right)$ & 5.5 & 11 & 14 \\
\hline \multirow[t]{4}{*}{ Ring-on-ring (Figure 2D) } & Thickness (d) & 30 & 48 & 75 \\
\hline & $\operatorname{Diameter}(\phi)$ & 262 & 420 & 657 \\
\hline & Radius to support $\left(\mathrm{R}_{\mathrm{S}}\right)$ & 125 & 200 & 312.5 \\
\hline & Radius of loading area $\left(\mathrm{R}_{l}\right)$ & 31.5 & 50 & 78 \\
\hline
\end{tabular}
center of the C1550 specimen to the support or the radius of the support ring for an RoR specimen; $r_{e}$ is the equivalent radius of the $\mathrm{C} 1550$ specimen,

TABLE 1 Dimensions of specimens used for 3 test methods ${ }^{17}$ 
$r_{e}=\sqrt{1.6 R_{l}^{2}+d^{2}}-0.675 d, R_{l}$ is the radius of the loaded area for a $\mathrm{C} 1550$ specimen or the radius of the loading ring for a RoR specimen.

Table 1 summarizes the dimensions of specimens used for the 3 test methods. Figure 7A summarizes tensile strength data, which were tabulated in $\mathrm{Zi}$ et al. ${ }^{17}$ As a result of proportional scaling for each type of specimen geometry, Figure 7A is transformed into Figure 7B.

Stress distribution inside the 3 types of specimens was calculated using 3-dimensional finite element method (FEM) to seek the values of the dimensionless coefficient $k$ according to Equation 26. The following parameters were used: Young's modulus E $=27264 \mathrm{MPa}$, Poisson's ratio $v=0.22$, Weibull modulus $\mathrm{m}=10$ and 8 . Note that the values of $m$ were obtained in Figure 7B. Equation 25a is adopted for linear regression of the experimental data in Figure 7B to estimate m. Figure 8 shows the examples of stress distribution in different specimens.

It was obtained that $\mathrm{k}_{4 \mathrm{~PB}}=0.686, \mathrm{k}_{\mathrm{C} 1550}=0.357$, $\mathrm{k}_{\mathrm{RoR}}=0.498$ for $\mathrm{m}=10$, and $\mathrm{k}_{4 \mathrm{~PB}}=0.637, \mathrm{k}_{\mathrm{C} 1550}=0.298$, $\mathrm{k}_{\mathrm{RoR}}=0.427$ for $\mathrm{m}=8$. According Equation 25c, Figure 7 $\mathrm{B}$ is transformed into Figure $7 \mathrm{C}, \mathrm{D}$ to correlate $\frac{1}{k^{m} \cdot V} \operatorname{Ln}\left[\frac{1}{(1-P)}\right]$ and nominal strength $\sigma_{\mathrm{N}}$ for $\mathrm{m}=10$ and $\mathrm{m}=8$, respectively. It can be seen that the strength data of all C1550 and RoR specimens in biaxial loading fall onto a master curve. However, the strength data of $4 \mathrm{~PB}$ specimens do not fit to the master curve. These trends are similar for either $\mathrm{m}=10$ (Figure $7 \mathrm{C}$ ) or $\mathrm{m}=8$ (Figure 7D).

\section{4 | DISCUSSIONS}

The 2 case studies of non-proportional size scaling are based on the assumptions of the maximum principal tensile stress fracture criterion $\left(\sigma_{e}=\sigma_{1}=\sigma \geq S\right)$ and stress state independence of the spatial distribution of microcracks. In both cases, Equation 25a is adopted for linear regression to estimate $\mathrm{m}$. As shown in Figure 6C, for the 2 loading modes of uniaxial tension and 3-point bending, which incur uniaxial stress states, all the strength data are synchronized onto a single master curve. While as shown in Figure 7C,D, the strength data of ASTM C1550 and RoR specimens, which are subjected to biaxial stress states, fall on a single master curve; while the strength data of the 4-point bending (4PB) specimens do not fall onto the master curve. This makes the strength data of the circular plates transferable between the 2 different loading modes (ASTM C1550 vs RoR tests). It also suggests that the maximum tensile stress fracture criterion and the assumption of a same spatial distribution
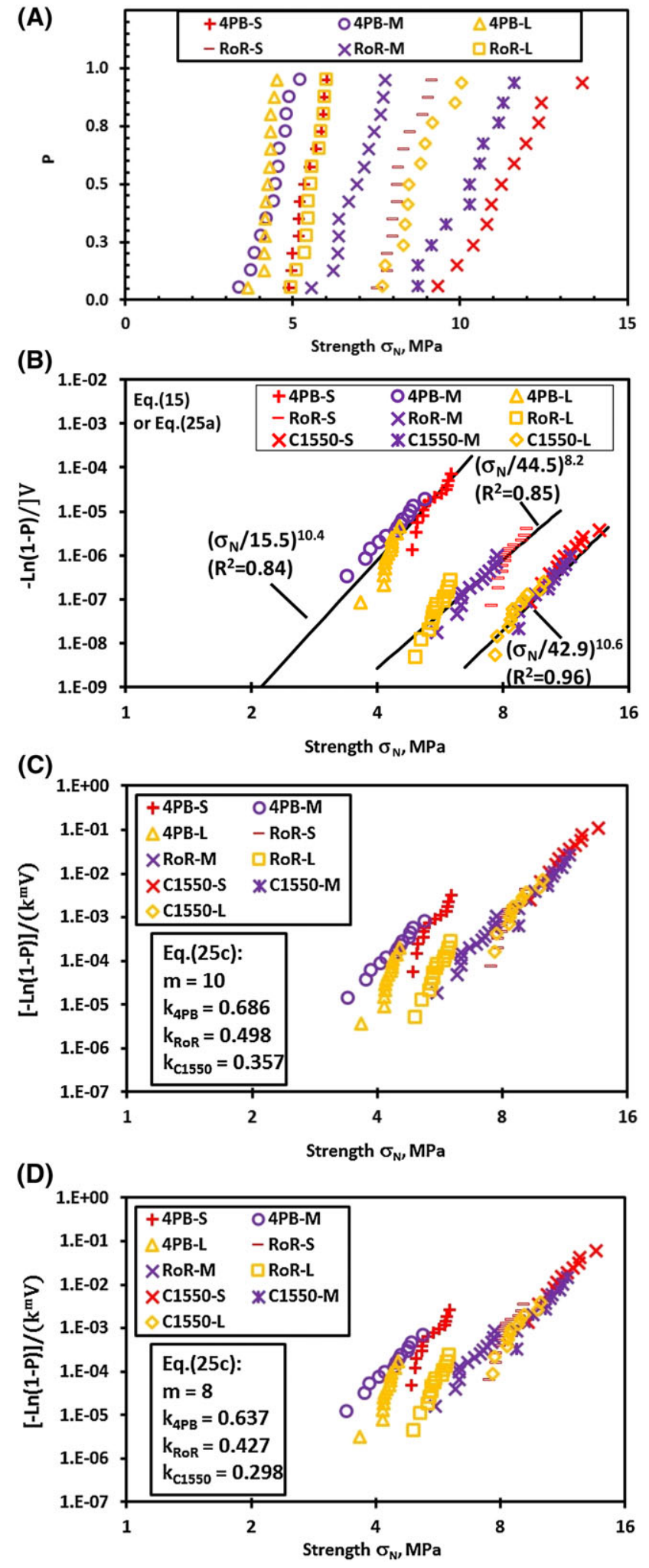

FIGURE 7 Non-proportional size scaling of strength in 4-point bending (4PB) prismatic beams, and ASTM C1550 (C1550) and ring-on-ring (RoR) biaxial bending of circular plates: A, raw experimental strength data ${ }^{13} ; \mathrm{B}$, proportional size scaling; $\mathrm{C}$, nonproportional size scaling with $\mathrm{m}=10$; and $\mathrm{D}$, non-proportional size scaling with $\mathrm{m}=8$ [Colour figure can be viewed at wileyonlinelibrary.com] 

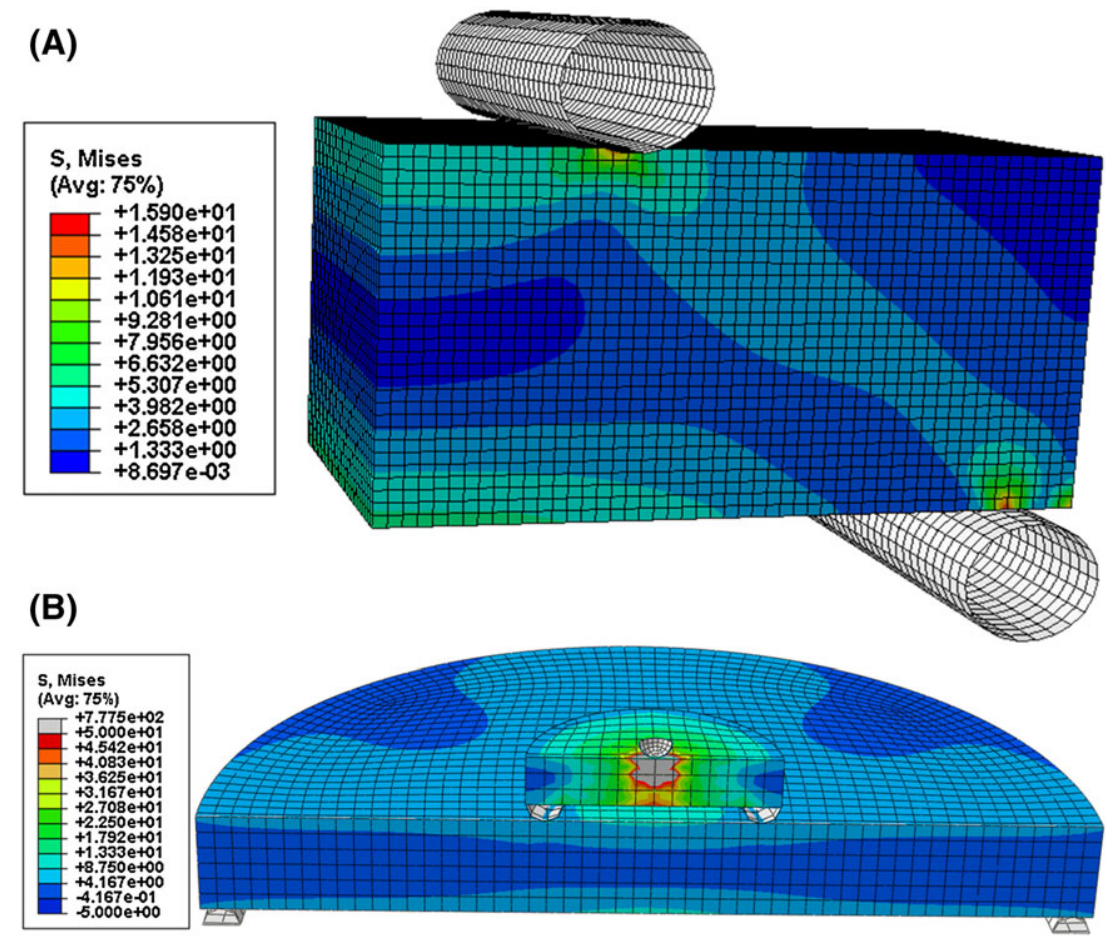

(B)

(C)

FIGURE 8 Elastic stress distribution in A, 4-point bending specimens, B, ring-onring specimens, and $\mathrm{C}$, ASTC $\mathrm{C} 1550$ specimens calculated by FEM [Colour figure can be viewed at wileyonlinelibrary.com]
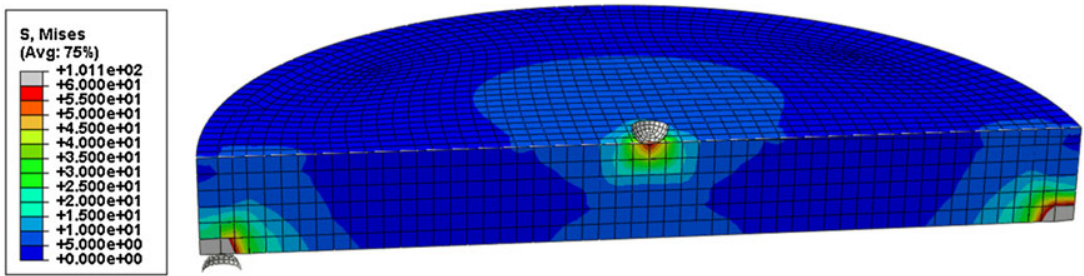

of microcracks are acceptable for the ASTM C1550 and RoR specimens. The deviation of the strength data of 4PB specimens from the master curve for those of ASTM C1550 and RoR specimens need to be explored further. As stated by $\mathrm{Zi}$, et al, ${ }^{17}$ an accurate analysis of the size effect in these 3-dimensional failures would be rather complicated and not easy to interpret. Here, an attempt is made to apply Equations 19 and 20 to analyze these data. One possibility is that the distribution of microcracks in uniaxial loading differs from that in

TABLE 2 Summary of estimated values of $m$ using maximum likelihood method and linear regression method of 3 types of specimens

\begin{tabular}{|c|c|c|c|c|c|}
\hline \multirow[b]{2}{*}{$\begin{array}{l}\text { Method of } \\
\text { Data Fitting }\end{array}$} & \multirow[b]{2}{*}{$\begin{array}{l}\text { Specimen } \\
\text { Size }\end{array}$} & \multirow{2}{*}{$\begin{array}{l}\text { Number of } \\
\text { Specimens } \\
\text { 4PB/C1550/ } \\
\text { RoR }\end{array}$} & \multicolumn{3}{|c|}{ Calibrated Value of $\mathbf{m}$} \\
\hline & & & 4PB & C1550 & RoR \\
\hline \multirow[t]{3}{*}{ Maximum likelihood } & $\mathrm{S}$ & $13 / 11 / 13$ & $13.7[9.4,20.3]^{\mathrm{a}}$ & $8.8[5.8,13.6]$ & $15.5[10.6,22.9]$ \\
\hline & M & $13 / 11 / 13$ & $9.5[6.5,14.0]$ & $10.9[7.2,16.8]$ & $11.3[7.7,16.6]$ \\
\hline & $\mathrm{L}$ & $13 / 11 / 13$ & $25.9[17.7,38.2]$ & $10.8[7.2,16.7]$ & $19.0[13.0,28.1]$ \\
\hline \multirow{2}{*}{ Linear regression $^{c}$} & M & $13 / 11 / 13$ & $9.4\left(R^{2}=0.989\right)$ & $9.9\left(R^{2}=0.930\right)$ & $11.1\left(R^{2}=0.959\right)$ \\
\hline & $\mathrm{L}$ & $13 / 11 / 13$ & $5.0\left(R^{2}=0.864\right)$ & $11.2\left(R^{2}=0.874\right)$ & $3.5\left(R^{2}=0.973\right)$ \\
\hline Equation $25 \mathrm{a}$ & $\mathrm{S}+\mathrm{L}+\mathrm{M}$ & $39 / 33 / 39$ & $10.4\left(R^{2}=0.835\right)$ & $10.6\left(R^{2}=0.959\right)$ & $8.2\left(R^{2}=0.848\right)$ \\
\hline
\end{tabular}

${ }^{\mathrm{a}} 13.7[9.4,20.3]$ refers to unbiased estimate of $\mathrm{m}=13.7$ with $90 \%$ confidence bounds 9.4 to 20.3 in the bracket.

${ }^{\mathrm{b}} R^{2}$ refers to the coefficient of determination.

${ }^{c}$ Linear regression is based on $\operatorname{Ln}\left[\operatorname{Ln}\left(\frac{1}{1-\mathrm{P}}\right)\right] \sim \operatorname{mLn}\left(\sigma_{N}\right)$ 
biaxial loading. However, at this point it is difficult to make a conclusive judgement, because this issue is confounded with the accuracy of the calibrated values of Weibull parameters. For example, the linear regression using Equation 25a yielded $\mathrm{m}=10.4,8.2,10.6$ for $4 \mathrm{~PB}$, C1550, and RoR specimens, respectively (Figure 7B). With the corresponding values of $k$, we get $\sigma_{0}=k \cdot \sigma_{u}=10.6$, 19.0, $15.3(\mathrm{MPa})$ in sequence. For comparison purposes, we also adopted maximum likelihood method and the linear regression method for each type of specimens at a given size (small, middle, large) to calibrate the value of $\mathrm{m}$, as summarized in Table 2. The maximum likelihood method and procedure follow ASTM Standard C1239-13: Standard Practice for Reporting Uniaxial Strength Data and Estimating Weibull Distribution Parameters for Advanced Ceramics. ${ }^{18}$ It can be seen that due to the limited amount of specimens at each size (13 for 4PB or RoR specimens and 11 for $\mathrm{C} 1550$ specimens), the values of $\mathrm{m}$ are much different $(\mathrm{m}=8.8-25.9$ by maximum likelihood method and $\mathrm{m}=3.5-17.8$ by linear regression method). The scatter of the calibrated values of $\mathrm{m}$ in different conditions is also reflected by the $90 \%$ confidence bounds in the case of maximum likelihood and the coefficient of determination $R^{2}$ in the case of linear regression. When the number of data points is below 30, it has a significant impact on the estimated value of $\mathrm{m}^{19}$ In other words, a good number of specimens is needed for each type to obtain better calibration of Weibull parameters.

\section{5 | SUMMARY AND CONCLUSIONS}

A procedure is proposed for non-proportional size scaling of the strength of concrete measured from specimens with different geometries in different loading modes. Proportional size scaling of strength is needed to determine the parameters of the weakest-link statistical model. Finite element analysis of stress distribution is conducted to calculate the coefficient of equivalent strength. Two case studies are conducted for validation. The following conclusions can be drawn:

(1). The non-proportional size scaling is feasible for the transference of strength data between specimens in a qualitatively same stress state. Specifically, the strength data from uniaxial tension and 3-point bending, both of which involve uniaxial stress states, are synchronized. The strength data from circular plates in the ring-on-ring and the ASTM C1550 test setups, both of which involve biaxial stress states, are also synchronized.

(2). The strength data from 4-point bending specimens (uniaxial stress state) and biaxial flexural discs are not synchronized onto a single master curve. The root cause is to be understood.

\section{ORCID}

G. Qian (D) http://orcid.org/0000-0003-0487-9780

W.-S. Lei (D) http://orcid.org/0000-0002-3003-2914

\section{REFERENCES}

1. Lei W-S, Yu Z. A statistical approach to scaling size effect on strength of concrete incorporating spatial distribution of flaws. Construct Build Mater. 2016;122:702-713.

2. Razavi SMJ, Ayatollahi MR, Berto F. A synthesis of geometry effect on brittle fracture. Eng Fract Mech. 2017;187:94-102. https://doi.org/10.1016/j.engfracmech.2017.10.022

3. Hu X, Guan J, Wang Y, Keating A, Yang S. Comparison of boundary and size effect models based on new developments. Eng. Fract. Mech. 2017;175:146-167.

4. Bazant ZP, Xi Y, Reid S. Statistical size effect in quasi-brittle structures: I. Is Weibull theory applicable? J Eng Mech. 1991;117(11):2609-2622.

5. Bazant ZP, Pang S-D. Activation energy based extreme value statistics and size effect in brittle and quasibrittle fracture. $J$ Mech Phys Solids. 2007;55(1):91-131.

6. Weibull W. A statistical theory of strength of materials. Roy. Swedish Inst. Eng. Res. 1939;1-45.

7. Batdorf, S. B. and Heinisch, H. L. Jr. (1978) Weakest link theory reformulated for arbitrary fracture criterion, $J$ Amer Ceram Soc $61,355-358,7-8$

8. Denzer R, Supancic P, Pascual J, Lube T. Fracture statistics of ceramics-Weibull statistics and deviations from Weibull statistics. Eng. Fract. Mech. 2007;74(18):2919-2932.

9. Le J-L, Falchetto AC, Marasteanu MO. Determination of strength distribution of quasibrittle structures from size effect analysis. Mech Mater. 2013;66:79-87.

10. Lei W-S. Evaluation of the basic formulations for the cumulative probability of brittle fracture with two different spatial distributions of microcracks. Fatigue Fract Eng Mater Struct. 2016;39(5):611-623.

11. Lei W-S. A framework for statistical modeling of plastic yielding initiated cleavage fracture of structural steels. Philos Mag. 2016;96(35):3586-3631.

12. Lei W-S. A generalized weakest-link model for size effect on strength of quasi-brittle materials. J Mater Sci. 2018;53(2):12271245.

13. Thiemeier $\mathrm{T}$, Bruckner-Foit A, Kolker $\mathrm{H}$. Influence of the fracture criterion on the failure probability of ceramic components. J. Am. Ceram Soc. 1991;74:48-52.

14. Lei W-S. A cumulative failure probability model for cleavage fracture in ferritic steels. Mech Mater. 2016;93:184-198.

15. Lei W-S. Fracture probability of a randomly oriented microcrack under multi-axial loading for the normal tensile stress criterion. Theor Appl Fract Mech. 2016;85:164-172. 
16. Griffith AA. The phenomena of rupture and flow in solids. Phil. Trans. Royal Soc. London A. 1921;221:163-198.

17. Zi, G., Kim, J., and Bazant, Z. P. (2014), Size effect on biaxial flexural strength of concrete. ACI Mater J 111,1-8, 3

18. ASTM standard C1239-13 (2013): Standard practice for reporting uniaxial strength data and estimating Weibull distribution parameters for advanced ceramics.

19. Davies IJ. Confidence limits for Weibull parameters estimated using linear least squares analysis. J Eur Ceram Soc. 2017;37(15):5057-5064.
How to cite this article: Qian G, Zhai J, Yu Z, Lei W-S, Wu W. Non-proportional size scaling of strength of concrete in uniaxial and biaxial loading conditions. Fatigue Fract Eng Mater Struct. 2018;41:1733-1745. https://doi.org/10.1111/ ffe. 12813 